\title{
TOPSIS extension for multi-objective supplier selection problem under price breaks.
}

\begin{abstract}
For supplier selection problem (SSP), if suppliers offer quantity discounts as well as buyer wants to buy multi product, SSP becomes more complicated. In order to solve the complicated problem, an integration of TOPSIS approach and multi-objective mixed integer linear programming (MOMILP) is used to define the optimum quantities among the selected suppliers. In this article, we also apply TOPSIS approach to solve the MOMILP problem. In this solution, TOPSIS minimizes the measure of distance, providing that the closest solution should have the shortest distance from the positive ideal solution (PIS) and the longest distance from the negative ideal solution (NIS) as well. Therefore, a q-dimensional objective space is reduced to a two dimensional space (PIS and NIS). Finally, a single objective function is then proposed as a suitable one to resolve the conflict between the new criteria (the shortest distance from the PIS and the longest distance from the NIS).
\end{abstract}

Keyword: Supplier selection; Multi-product; Quantity discount; TOPSIS; MOMILP. 